\title{
Magnetic field near the central region of the Galaxy: rotation measure of extragalactic sources
}

\author{
S. Roy ${ }^{1}$, A. Pramesh Rao ${ }^{2}$, and R. Subrahmanyan ${ }^{3}$ \\ 1 ASTRON, PO Box 2, 7990 AA Dwingeloo, The Netherlands \\ e-mail: roy@astron.nl \\ 2 National Centre for Radio Astrophysics (TIFR), Pune University Campus, Post Bag No. 3, Ganeshkhind, Pune 411 007, India \\ e-mail: pramesh@ncra.tifr.res.in \\ Raman Research Institute, C. V. Raman Avenue, Sadashivanagar, Bangalore 560 080, India
}

Received 28 September 2006 / Accepted 9 October 2007

\section{ABSTRACT}

\begin{abstract}
Aims. We determine the properties of the Faraday screen and the magnetic field near the central region of the Galaxy.
Methods. We measured the Faraday rotation measure (RM) towards 60 background extragalactic source components through the $-6^{\circ}<l<6^{\circ},-2^{\circ}<b<2^{\circ}$ region of the Galaxy using the 4.8 and $8.5 \mathrm{GHz}$ bands of the ATCA and VLA. Here we use the measured RMs to estimate the systematic and the random components of the magnetic fields.

Results. The measured RMs are found to be mostly positive for the sample sources in the region. This is consistent with either a large scale bisymmetric spiral magnetic fields in the Galaxy or with fields oriented along the central bar of the Galaxy. The outer scale of the RM fluctuation is found to be about $40 \mathrm{pc}$, which is much larger than the observed RM size scales towards the non thermal filaments (NTFs). The RM structure function is well-fitted with a power law index of $0.7 \pm 0.1$ at length scales of 0.3 to $100 \mathrm{pc}$. If Gaussian random processes in the ISM are valid, the power law index is consistent with a two dimensional Kolmogorov turbulence. If there is indeed a strong magnetic field within $\sim 1^{\circ}$ (radius $150 \mathrm{pc}$ ) from the GC, the strength of the random field in the region is estimated to be $\sim 20 \mu \mathrm{G}$.

Conclusions. Given the highly turbulent magnetoionic ISM in this region, the strength of the systematic component of the magnetic fields would most likely be close to that of the random component. This suggests that the earlier estimated milliGauss magnetic field near the NTFs is localised and does not pervade the central $300 \mathrm{pc}$ of the Galaxy.
\end{abstract}

Key words. ISM: magnetic fields - Galaxy: center - techniques: polarimetric

\section{Introduction:}

Magnetic fields are widely recognised as playing an important role in the evolution of supernova remnants, in star formation, overall structure of ISM, cosmic ray confinement and nonthermal radio emission. This is especially true in central region of the Galaxy, where magnetic fields could be strong enough to be significant in the dynamics and evolution of the region (Beck et al. 1996). A relatively high systematic magnetic field in the Galactic centre (GC) region was believed to be responsible for the creation and maintenance of the unique non thermal filaments (NTFs) (Morris et al. 1996, and references therein). Therefore, it is important to measure the magnetic-field geometry and strength near the central part of the Galaxy.

Other than the central $200 \mathrm{pc}$ of the GC, no systematic study has been made in the past to measure the magnetic fields in the inner $5 \mathrm{kpc}$ region of the Galaxy (Davidson 1996). Recently, Brown et al. (2007) have surveyed the 4th quadrant of the Galaxy up to $l=358^{\circ}$ through Faraday RMs, but their observations do not target the central kpc of the Galaxy. The earlier estimates of magnetic fields within the central $200 \mathrm{pc}$ of the Galaxy were based mainly on observations of the non-thermal filaments, and the measured Faraday rotation measure (RM) towards these NTFs were found to be $\sim 1000 \mathrm{rad} \mathrm{m}^{-2}$ (Yusef-Zadeh \& Morris 1987b; Anantharamaiah et al. 1991; Gray et al. 1995; Yusef-Zadeh et al. 1997; Lang et al. 1999b). Since magnetic pressure in these NTFs appears to overcome turbulent
ISM pressure (otherwise, the NTFs would have bent due to interaction with molecular clouds), Yusef-Zadeh \& Morris (1987a) derived a magnetic-field strength of about 1 milliGauss within these NTFs. Moreover, the high magnetic field in the region is required to be ubiquitous. Otherwise, in regions where there is no molecular cloud around NTFs, magnetic pressure within these structures would be much higher than outside. This will cause the NTFs to expand at Alfven speed and decay at a time scale ( $\sim 300$ years) that is likely to be much less than their formation time scales (Morris 1998). This implies that if the NTFs are static structures, the magnetic field in the region must be ubiquitous (Morris et al. 1996).

Earlier measurements of direction of magnetic fields within the NTFs have shown it to be oriented along their length. Since all the well known NTFs found within a degree of the GC are oriented almost perpendicular to the Galactic plane, it suggests the field lines in the surrounding ISM are also perpendicular to the Galactic plane (Morris et al. 1996, and the references therein). In addition, the NTF Pelican (G358.85+0.47) (Lang et al. 1999a) located about a degree from the GC is found to be almost parallel to the Galactic plane. This indicates that the field lines change their orientation from being perpendicular to parallel to the plane beyond a degree from the GC, which is typically observed in the rest of the Galaxy. However, we note that if the NTFs are manifestations of peculiar local environments (Shore \& Larosa 1999), inferences drawn from these observations can be 
misleading. With the recent discovery of many new fainter filamentary structures in the GC region oriented quite randomly with the Galactic plane (Nord et al. 2004), serious doubts are cast on the orientation of the magnetic field and its ubiquitous nature near the GC.

Zeeman splitting of spectral lines can directly yield the magnetic field in a region. However, this method is known to be sensitive to small-scale fields, and therefore high magnetic field strengths in a small region anywhere along the line-of-sight (LOS) can indicate a high magnetic field, which is not representative of the average. Therefore, past estimates of milliGauss magnetic fields based on Zeeman splitting (Schwarz \& Lasenby 1990; Killeen et al. 1992; Yusef-Zadeh et al. 1996, 1999) of HI or $\mathrm{OH}$ lines towards the GC could have resulted from local enhancement of field (e.g., near the cores of high-density molecular clouds). To measure any systematic magnetic field in the region, it is necessary to use an observational technique that is sensitive to large-scale fields. To avoid manifestations of favourable local environments, Galactic objects should not be used for this purpose.

Faraday rotation measure is the integrated LOS magnetic field weighted by the electron density

$R M=0.81 \times \int n_{\mathrm{e}} B_{\|} \mathrm{d} l$,

where, $R M$ is rotation measure expressed in $\operatorname{rad~m}^{-2}, n_{\mathrm{e}}$ is the electron density expressed in $\mathrm{cm}^{-3}, B_{\|}$is the LOS component of the magnetic field in $\mu \mathrm{G}$, and the integration is carried out along the LOS, with distance expressed in parsec. If a model for the electron density is available, observations of RM towards the extragalactic sources seen through the Galaxy can be used to estimate the average magnetic fields in the ISM. A large number of studies of the Galactic magnetic fields have already been made using RM towards the extragalactic sources Simard-Normandin \& Kronberg (1980); Frick et al. (2001); Clegg et al. (1992). Similar studies have also been carried out towards pulsars (Rand \& Kulkarni 1989; Rand \& Lyne 1994; Han $\&$ Qiao 1994). These studies have shown that there is one field reversal within and one outside the solar circle, while two more reversals have been suggested by Han et al. (1999). These reversals could be explained by invoking either the bisymmetric spiral model (Simard-Normandin \& Kronberg 1980; Han et al. 1999) or a ring model, where the direction of the field lines reverses in each ring (Rand \& Kulkarni 1989). However, there has been no systematic observation of RM towards extragalactic sources seen through the GC region.

We systematically studied RM properties of 60 extragalactic sources seen through the central $-6^{\circ}<l<6^{\circ},-2^{\circ}<b<2^{\circ}$ region of the Galaxy. The angular scale over which the magnetoionic medium is coherent near the NTFs has been estimated as $\sim 10^{\prime \prime}$ (Gray et al. 1995; Yusef-Zadeh et al. 1997). Therefore, to avoid any beam depolarisation introduced by the ISM of the Galaxy, our observations were made with the higher resolution configurations of these telescopes, so that the synthesised beam sizes are considerably smaller than the coherence scale length of the Faraday screen near the GC. Preliminary results of these observations were published earlier in Roy et al. (2003) and Roy (2004). In Roy et al. 2005 (2005, henceforth Paper I), we described the sample sources, the observations and data analysis and then determined their spectral indices, polarisation fraction, $\mathrm{RM}$, and the direction of their intrinsic magnetic field. In this paper, we interpret the RM observations. In Sect. 2, we provide a graphic representation of the measured RMs (see Paper I), while

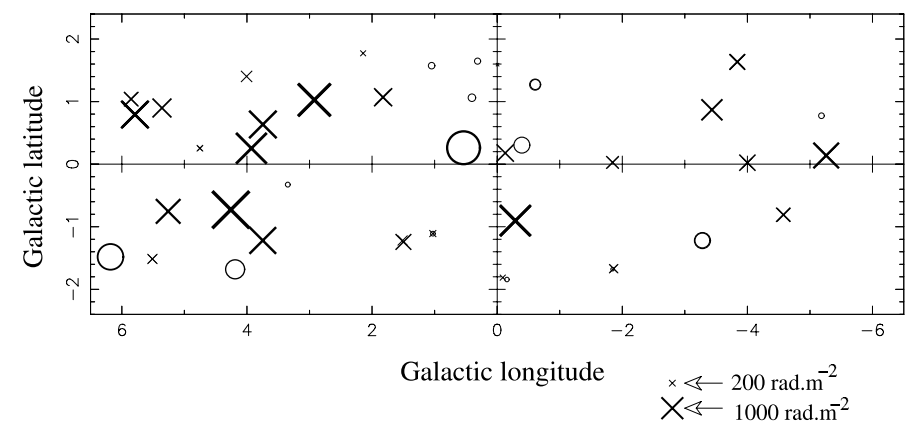

Fig. 1. Measured Faraday RMs towards the polarised sources in Galactic co-ordinate. The positive values are indicated by "cross (X)" and negative values by "circle $(\mathrm{O})$ ". Size of the symbols increase linearly with $|R M|$.

interpretation of the results is described in Sect. 3. The conclusions are presented in Sect. 4.

\section{Results}

\subsection{Features in the Faraday screen near the GC}

In Fig. 1, we plot RMs of 60 polarised components (including 2 secondary calibrators), which conform to the criteria given in Paper I (i.e., reduced $\chi^{2}$ of the polarisation angle vs. frequency fit less than or equal to 4.6 , depolarisation fraction between 4.8 and $8.5 \mathrm{GHz}$ higher than or equal to 0.6 and the source is outside the Galaxy). This figure shows our measured RMs divided into four quadrants according to the signs of Galactic longitude and latitude. In the rest of this paper, we define quadrant A when $l$ and $b$ are both positive, quadrant $\mathrm{B}$ when $l$ is negative but $b$ is positive, quadrant $\mathrm{C}$ when both $l$ and $b$ are negative and quadrant $\mathrm{D}$ when $l$ is positive but $b$ is negative. The region is dominated by positive RMs, as observed towards most of the sources in both positive and negative Galactic longitude. The observed RMs towards sources with $|b| \leq 1.5^{\circ}$ are quite high $\sim 1000 \mathrm{rad} \mathrm{m}^{-2}$. Such high RMs have been measured towards extragalactic sources at low Galactic latitudes $\left(45^{\circ}<l<93^{\circ}\right.$, and $\left.|b|<5^{\circ}\right)$ by Clegg et al. (1992) and are due to passage of radio wave through large path lengths along interstellar medium. These results are consistent with positive RMs observed near $l=-5^{\circ}$ by Brown et al. (2007), which lies near the edge of their survey.

In general, magnetoionic media responsible for the RMs have structures at different length scales. Reversal of sign of the RM over angular scales of a few degrees shows the existence of a random component of the magnetic field. We explore this through the structure function analysis of the RMs and then identify systematic features in the data.

\subsubsection{The structure function analysis of RMs}

Variations in RM over an angular scale of $\Delta \theta$ can be described by the RM structure function $D(\Delta \theta)=\left\langle[R M(\theta)-R M(\theta+\Delta \theta)]^{2}\right\rangle$. The structure function is measured by computing the expectation value of the squared differences of the RM among all pairs of sources within a particular range of angular separation $(\Delta \theta)$. We binned the data with $\Delta \theta$ from $0.0^{\circ}$ to $0.005^{\circ}, 0.005^{\circ}$ to $0.1^{\circ}$, $0.1^{\circ}$ to $0.33^{\circ}, 0.33^{\circ}$ to $0.6^{\circ}$, and then up to $1.0^{\circ}$ in bin widths of $0.2^{\circ}$. From $1.0^{\circ}$ to $4.0^{\circ}$, we binned angular separations with bin widths of $0.5^{\circ}$, and from $4.0^{\circ}$ to $10^{\circ}$ a single bin was used. The RM structure function in each bin is plotted at the location of the median angular separation of sources in that bin in Fig. 2. 
Table 1. Rotation measure of sources binned along Galactic longitude.

\begin{tabular}{|c|c|c|c|c|c|c|c|}
\hline $\begin{array}{l}\text { Bin } \\
\text { No. }\end{array}$ & $\begin{array}{l}\text { Range } \\
\text { in } l \\
\text { (deg) }\end{array}$ & $\begin{array}{l}\text { No. of } \\
\text { sources }\end{array}$ & $\begin{array}{c}\text { Mean } \\
\text { RM } \\
\left(\mathrm{rad} \mathrm{m}^{-2}\right)\end{array}$ & $\begin{array}{c}\text { Rms on } \\
\text { mean RM } \\
\left(\mathrm{rad} \mathrm{m}^{-2}\right)\end{array}$ & $\begin{array}{l}\text { No. of } \\
\text { sources } \\
\text { flagged }\end{array}$ & $\begin{array}{c}\text { Mean RM } \\
\text { after sources } \\
\text { flagged } \\
\left(\mathrm{rad} \mathrm{m}^{-2}\right)\end{array}$ & $\begin{array}{c}\text { Rms on mean } \\
\text { RM after } \\
\text { flagging } \\
\left(\mathrm{rad} \mathrm{m}^{-2}\right)\end{array}$ \\
\hline 1 & -6.0 to -3.6 & 5 & 534 & 183 & $\overline{0}$ & 534 & 183 \\
\hline 2 & -3.6 to -1.2 & 4 & 281 & 305 & 0 & 281 & 305 \\
\hline 3 & -1.2 to 1.2 & 11 & -3 & 201 & 4 & -82 & 52 \\
\hline 4 & 1.2 to 3.6 & 5 & 577 & 277 & 0 & 577 & 277 \\
\hline 5 & 3.6 to 6.0 & 12 & 827 & 193 & 1 & 959 & 154 \\
\hline
\end{tabular}

Table 2. Rotation measure of sources binned along Galactic latitude.

\begin{tabular}{cccccccc}
\hline \hline $\begin{array}{c}\text { Bin } \\
\text { No. }\end{array}$ & $\begin{array}{c}\text { Range } \\
\text { in } l\end{array}$ & $\begin{array}{c}\text { No. of } \\
\text { sources }\end{array}$ & $\begin{array}{c}\text { Mean } \\
\text { RM } \\
(\mathrm{deg})\end{array}$ & $\begin{array}{c}\text { Rms on } \\
\text { mean RM }\end{array}$ & $\begin{array}{c}\text { No. of } \\
\text { sources } \\
\text { flagged } \\
\left(\mathrm{rad} \mathrm{m}^{-2}\right)\end{array}$ & $\begin{array}{c}\text { Mean RM } \\
\text { after sources } \\
\text { flagged } \\
\left(\mathrm{rad} \mathrm{m}^{-2}\right)\end{array}$ & $\begin{array}{c}\text { Rms on mean } \\
\text { RM after } \\
\text { flagging } \\
\left(\mathrm{rad} \mathrm{m}^{-2}\right)\end{array}$ \\
\hline 1 & -2.0 to -1.2 & 8 & 43 & 246 & 1 & -129 & 203 \\
2 & -1.2 to -0.4 & 5 & 1009 & 304 & 0 & 1009 & 304 \\
3 & -0.4 to 0.4 & 9 & 295 & 267 & 1 & 478 & 220 \\
4 & 0.4 to 1.2 & 9 & 766 & 196 & 0 & 766 & 196 \\
5 & 1.2 to 2.0 & 7 & 107 & 130 & 0 & 107 & 130 \\
\hline
\end{tabular}

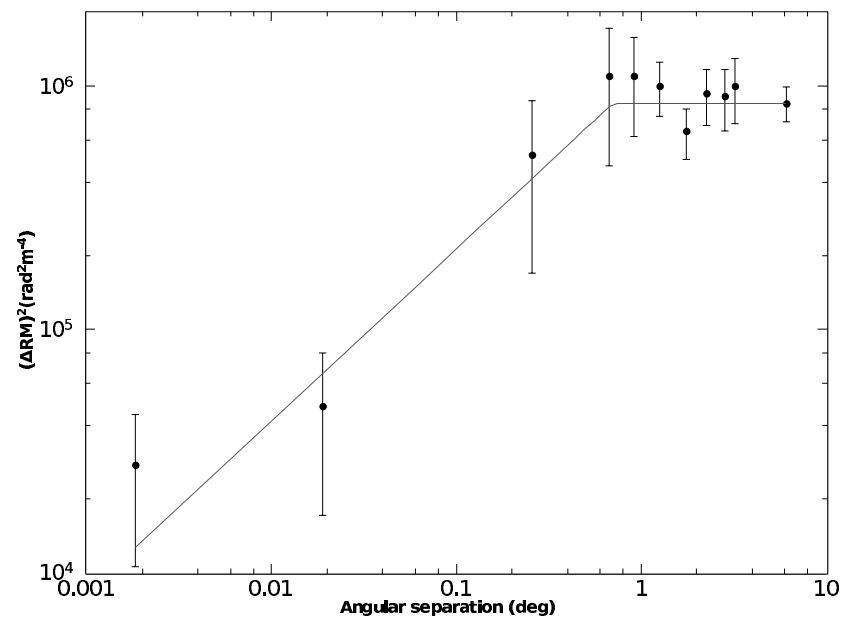

Fig. 2. The structure function of the measured RMs. See text for the fit.

The errorbars in the plot were estimated by a statistical method called "Bootstrap" (Efron 1976). Figure 2 shows that the structure function appears to saturate at beyond $\sim 0.7^{\circ}$; therefore, we fitted a power law $f(\Delta \theta)=A \times \Delta \theta^{n}$ for $\Delta \theta \leq 0.7^{\circ}$, and then $f(\Delta \theta)$ is held fixed at its value for $\Delta \theta=0.7^{\circ}$. The function $[f(\Delta \theta)]$ is well-fitted to the data (reduced $\chi^{2} 0.47$ ), and is shown in Fig. 2. From the fit, $A$ is found to be $1.1 \pm 0.1 \times 10^{6}$, and the power law index $(n)$ is $0.7 \pm 0.1$.

The outer scale of the structure function is defined as the length scale at which the structure function attains half of its maximum value (Rickett 1988). From the data, we estimate the outer scale to be about $0.3^{\circ}$ or $40 \mathrm{pc}$ for the screen if located at the distance of GC, $8.0 \mathrm{kpc}$ away. This indicates the RMs of sources lying within an angular distance of $<0.3^{\circ}$ are likely to be correlated. Therefore, while determining statistical quantities in this paper, we ensure independent measurements by considering the RMs of source components located at least beyond $0.2^{\circ}$ from each other. There are a total of 38 source components that conforms to this criterion.

Given the extreme conditions in the ISM close to the GC, statistical properties of the medium in this region could be different from that of its immediate surroundings. To check for any change in outer scale of RM for sources seen within $1.1^{\circ}$ $(\sim 150 \mathrm{pc})$ of the GC, we carried out the above analysis for 6 sources seen through the region. The structure function of these sources with angular separations less than $1.1^{\circ}$ is $9.5 \pm 5.5 \times$ $10^{5} \mathrm{rad} \mathrm{m}^{-2}$, and is $3.8 \pm 1.6 \times 10^{6} \mathrm{rad} \mathrm{m}^{-2}$ for sources with angular separations between $1.1^{\circ}$ to $2.1^{\circ}$. This shows the RMs changed by more than 1.7 times the effective error, indicating that the structure function of RMs is not saturated for angular separations of less than a degree ( $>90 \%$ confidence). While the significance of this result is not very high due to the small number of sources in the sample, we adopt the simplest model and assume the outer scale in the inner $1.1^{\circ}$ region is comparable to $40 \mathrm{pc}$ determined from the full sample.

\subsubsection{Large-scale pattern in the RMs data}

In Fig. 1, we notice the dominance of sources with positive RMs. Following the criteria given above for sources with uncorrelated RMs, we find the mean RM from the data to be $413 \pm$ $115 \mathrm{rad} \mathrm{m}^{-2}$, and the median is $476 \mathrm{rad} \mathrm{m}^{-2}$. The mean RM in quadrant A of Fig. 1 is $488 \pm 204,396 \pm 294$ in quadrant B, $354 \pm 170$ in $C$ and $350 \pm 323$ in D. To study the systematic behaviours of this large-scale field, we divided the observed region in several bins along the Galactic longitude and latitude such that a reasonably large number of sources remain in each bin to yield meaningful statistical properties (mean, rms) of RMs in these bins. Therefore, we selected 5 bins along the Galactic longitude, each $2.4^{\circ}$ wide resulting in $\sim 8$ sources per bin, and the resulting statistical properties of RMs from each of these bins is tabulated in Table 1. Similarly, in Table 2 we have tabulated statistical properties of RMs along Galactic latitude divided in 5 bins of width $0.8^{\circ}$. Average RMs along Galactic longitude and latitude are plotted in Fig. 3 (shown with solid error-bars) and Fig. 4, respectively. Figure 3 shows that the average RM of sources located within $|l|<1^{\circ}$ is significantly less than that of sources located beyond $|l|>3^{\circ}$.

To remove possible small-scale variations in RM due to LOS HII regions or supernova remnants (Mitra et al. 2003), thereby getting a clearer picture of the large-scale field in the region, we 


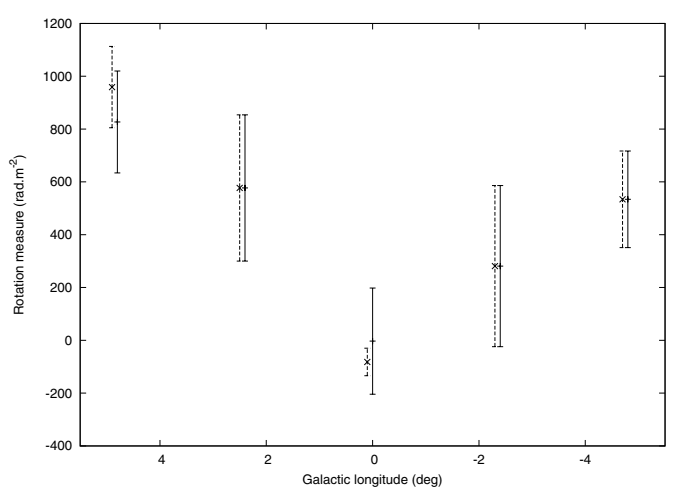

Fig. 3. Plot of RMs as a function of Galactic longitude. The values shown are averaged over 2.4 degree bins in longitude. Data points with solid error bars are before flagging, and data after flagging are displayed by dashed errorbars with axis shifted by $-0.1^{\circ}$ to what is displayed along the axis at the bottom. Details of flagging are described in the text.

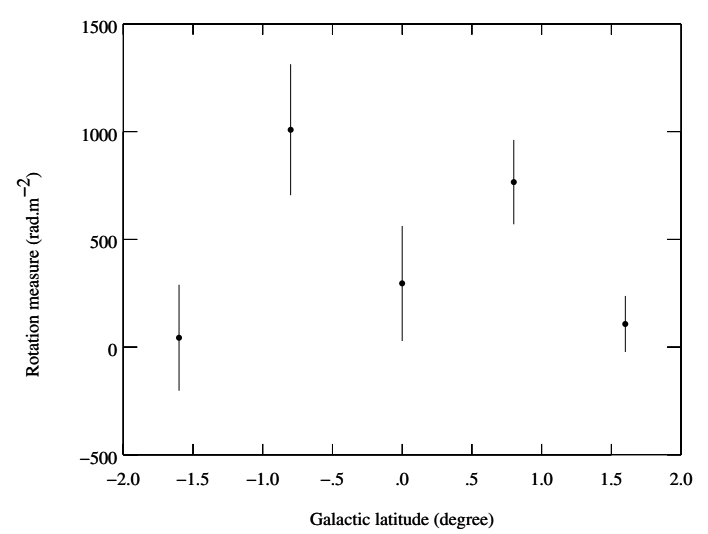

Fig. 4. Plot of RMs as a function of Galactic latitude. The values shown are averaged over 0.8 degree bins.

used the following method. We estimate the mean and rms RM of sources located in each bin in Table 1. If measured RM of any source within a bin deviates from the mean in that bin beyond 1.7 times the rms ( $\leq 10 \%$ probability for Gaussian distributed errors), that RM is rejected (flagged) and the mean and rms RM in that bin is recomputed. This process is repeated till there is no source outside the flag limit. Since there are only a few sources per bin, flagging the highly deviant data points in a bin would reduce the measured rms as compared to the real $\mathrm{rms}$ in the data. However, in a majority of cases, it results in a drop in measured rms of only $\sim 25 \%$, and the probability of a decrease in rms noise by a factor of 4 is $<10 \%$. Five out of 38 sources were rejected as a result of the flagging by this method, and the minimum number of sources in any of the bins after flagging were 4 . The resulting mean and rms RM values in each bin are tabulated in Table 1. We note that most of the flagging was in the central bin with $-1.2^{\circ}<l<1.2^{\circ}$, where 4 out of 11 sources were flagged, and the rms RM of sources decreased by almost a factor of 5 after flagging ( $\sim 5 \%$ probability with Gaussian random noise), indicating a significant small-scale structure (non-Gaussian errors) in the Faraday screen towards this region. The resulting distribution of average RM is plotted in Fig. 3 using dashed errorbars, and to make the symbols visible, the $\mathrm{X}$-axis of this plot is shifted by $-0.1^{\circ}$ from what is shown at the bottom. This shows that the average RM tends to zero near $l=0^{\circ}$. We applied the same procedure for RMs of sources located in each of the bins in Table 2, and the resulting mean and rms RM values after flagging in each bin are tabulated there. No significant change in mean or rms $\mathrm{RM}$ is noticed after flagging in this case.

\section{Discussion}

In the previous section we identified a largely positive RM towards background sources, the correlation length of which is about 40 pc. The RMs averaged in bins along the Galactic longitude was found to decrease near $l=0^{\circ}$. In this section, we identify the location and properties of the Faraday screen that is responsible for the above. Then, using a plausible model of the electron density distribution near the GC, we investigate the nature of the magnetic fields (comprised of systematic and random components) in the region.

\subsection{Location of the Faraday screen}

\subsubsection{Small-scale structures in the Faraday screen and intrinsic RMs of sources}

Differences in RMs seen along different LOS could occur from either (i) a geometrical effect or (ii) change in the property of the Faraday screen. The structure function due to a perfectly uniform Faraday screen will have a measurable geometrical component simply because of the change in the LOS component of the field with change in the $l$ and $b$. An observer embedded in an extended homogeneous medium with uniform magnetic field approaching from an arbitrary angle $\theta_{0}$ sees a rotation measure $R M_{0} \cos \left(\theta-\theta_{0}\right)$ (Clegg et al. 1992), where $R M_{0}$ is the $R M$ towards $\theta_{0}$. However, over the observed longitude range, the contribution from the variation in the "cosine" term is much less than what is observed in Fig. 2. Therefore, we do not consider the geometrical effect any further.

The measured RMs towards the sources could have a significant intrinsic contribution from a magnetoionic medium local to the sources. However, in this case, intrinsic RMs towards different sources will be uncorrelated. Consequently, differences in RMs for unrelated sources will persist regardless of their location on the sky plane, and will not approach zero when their angular separation tends to zero. However, in Fig. 2, we find the RM structure function tends to zero at zero angular separation and increases smoothly with source angular separations. This shows intrinsic RMs can be neglected, and the measured RMs have an interstellar origin (plasma turbulence) within our Galaxy.

\subsubsection{RM contribution from the Galactic disk}

To explain the observed magnetic field orientation in our Galactic disk, two models of magnetic fields, the ring (Rand \& Kulkarni 1989) and the bisymmetric spiral (Simard-Normandin \& Kronberg 1980) are widely used. However, both of these models predict that the LOS RM contribution from the Galactic disk is quite small when $|l| \ll 1 \mathrm{rad}$. Ionised gas located close to us along the LOS could, however, produce a bias on a large angular scale to the observed RMs. In this case, nearby pulsars seen towards GC will also show such correlated RMs. We searched for pulsar RMs located in our survey region from ATNF Pulsar Catalogue (Manchester et al. 2005) and found 7 pulsars with measured RMs that are located closer than the GC. Their distance as estimated from their dispersion measure (Taylor \& Cordes 1993) varies from $1.5 \mathrm{kpc}$ to $7.7 \mathrm{kpc}$ with a median value of $3.5 \mathrm{kpc}$. Mean RM of these sources is $-7 \pm 46 \mathrm{rad} \mathrm{m}^{-2}$. Since 
the mean RM is quite small, any Faraday screen affecting our sample has to be located at least beyond the median distance of these pulsars. Moreover, the linear size of an object at this median distance of $3.5 \mathrm{kpc}$ with angular size of our survey will be $\sim 300$ pc. Objects known to produce significant RMs (e.g., HII regions, supernova remnants) are typically much smaller than the above size scale. Therefore, no single nearby object has significantly biased the RMs, so we believe the central few kpc region of the Galaxy is responsible for the observed RMs.

\subsection{Magnetic field near the GC}

Faraday rotation being the LOS integral of the product of the magnetic field with the electron density, changes in electron density or the magnetic field strengths or a change in the direction of the magnetic field vector can contribute variations in the observed RM. To separate the contribution of these effects, we first discuss the available models of the electron density distribution and then discuss the large-scale magnetic field near the central region of the Galaxy.

\subsubsection{Electron density distribution near the GC and strength of the large-scale magnetic field}

The electron density of the ISM is believed to increase towards the central region of the Galaxy. Different electron density models are invoked for the inner Galaxy, central kpc, and the central $100 \mathrm{pc}$ of the Galaxy, which are discussed below.

Taylor \& Cordes (1993) modelled electron density distribution in the Galaxy and included an inner Galactic component that is considered a ring at a distance of $\sim 4 \mathrm{kpc}$ from the GC. However, their model does not include a GC component. Over the central few degrees of the GC, Bower et al. (2001) carried out VLBA observations of 3 extragalactic sources and report a region of enhanced scattering covering $\gtrsim 5^{\circ}$ in longitude and $\leq 5^{\circ}$ in latitude. The measured scattering diameters correspond to about $\sim 300$ milli-arcsec at $1 \mathrm{GHz}$, which is $1.5-6$ times the prediction from the Taylor \& Cordes (1993) model. Using scatter broadening of $\mathrm{OH}$ masers in the vicinity of $\mathrm{OH} / \mathrm{IR}$ stars, van Langevelde et al. (1992) showed that there is a region of high scattering within $30^{\prime}$ of the GC. From free-free absorption measurements, they suggest the scattering region is at a distance of more than $850 \mathrm{pc}$ from the GC. Using a likelihood analysis, Lazio \& Cordes (1998) claim a "hyperstrong" scattering screen $\left(n_{\mathrm{e}} \sim 10 \mathrm{~cm}^{-3}\right)$ of the same angular extent $\left(30^{\prime}\right)$ towards the $\mathrm{GC}$, but estimated the distance to this screen to be $133_{-80}^{+200} \mathrm{pc}$ from the GC. This model predicts a scattering diameter for extragalactic sources to be an order of magnitude higher than what is observed by Bower et al. (2001). However, the extragalactic source G359.87+0.18 (Lazio et al. 1999) is seen through the "hyperstrong scattering" region, but its scattering size is an order of magnitude lower than predicted from the "hyperstrong scattering" model. This indicates the screen is patchy Lazio et al. (1999). An improved version of Taylor \& Cordes (1993) model has been published by Cordes \& Lazio (2002), where contribution from a GC component corresponding to the contribution from the central 30' region of the Galaxy (Lazio \& Cordes 1998) has been added. However, it does not include any contribution from the enhanced scattering region observed by Bower et al. (2001). In our observations, all the objects barring one $(\mathrm{G} 359.87+0.18)$ are seen through the region of enhanced scattering observed by Bower et al. (2001). Therefore, we used their observations to estimate electron density, which will be used in the rest of the paper. If we assume the turbulence scale length of this screen to be the same as that of the inner Galaxy component of Taylor \& Cordes (1993), Bower et al. (2001) scattering measure imply an electron density of about $0.4 \mathrm{~cm}^{-3}$. This is twice of what is estimated from the Taylor \& Cordes (1993) model for the inner Galaxy. The corresponding dispersion measure from the inner $2 \mathrm{kpc}$ of the Galaxy is $800 \mathrm{pc} \mathrm{cm}^{-3}$. From the Cordes \& Lazio (2002) model, we also estimate the dispersion measure from the rest of the Galaxy along the LOS passing about a degree away from the GC, which is found to be $800 \mathrm{pc} \mathrm{cm}^{-3}$. Therefore, the total dispersion measure towards the inner kpc of the Galaxy is $\sim 1600 \mathrm{pc} \mathrm{cm}^{-3}$, and half of the total dispersion measure originates from the inner Galaxy component. It should be noted that at present the dispersion measure of the inner Galaxy component is uncertain by factor of a few. Using the above-mentioned dispersion measure of $800 \mathrm{pc} \mathrm{cm}^{-3}$ for the central $2 \mathrm{kpc}$ of the Galaxy and mean RM of $413 \mathrm{rad} \mathrm{m}^{-2}$ (Sect. 2) in Eq. (1), the mean LOS magnetic field is estimated to be $0.6 \mu \mathrm{G}$. As this is an LOS average, it should be treated as a lower limit.

\subsubsection{Geometry of the large scale azimuthal magnetic field}

In the presence of various turbulent processes in the GC, any unravelling of the large-scale field orientation needs to be performed statistically, and here we consider possible models to explain the results (Sect. 2).

(i) Magnetohydrodynamic model:

Uchida et al. (1985) proposed this model to explain the Galactic Centre Lobes (GCL), which are a pair of limb-brightened radio structures of several hundred parsecs extending from Galactic plane towards positive Galactic latitudes (Sofue \& Handa 1984) and seen within the central $1^{\circ}$ of the Galaxy. They carried out non-steady axisymmetric magnetohydrodynamic simulations in which the magnetic field is assumed to be axial at high Galactic latitudes. However, due to the differential rotation of dense gas near the Galactic plane, the field acquires a component along this plane. This model predicts an LOS field in quadrants A and C towards the observer (positive RM), and away from the observer in quadrants B and D. Novak et al. (2003) find the signs of the measured RMs towards the known NTFs to be consistent with the above prediction. From our observations, the estimated mean RM towards sources seen through quadrants A and C is $432 \pm$ $133 \mathrm{rad} \mathrm{m}^{-2}$ and $379 \pm 217 \mathrm{rad} \mathrm{m}^{-2}$ through quadrants B and D. Positive RMs in all the quadrants are inconsistent with their prediction. However, our sources are observed over a significantly bigger region around the GC than the NTFs are seen, and the results do not match the prediction of this model.

(ii) Ring model:

According to this model, magnetic field lines in a galaxy are oriented along circular rings in the galactic plane. As discussed in Rand \& Kulkarni (1989), such a geometry arises in galactic dynamo models of the field, in which a symmetric azimuthal mode is dominant (e.g., Krause 1987). Theories involving a primordial origin of magnetic field also claim to be able to produce ring fields, but only in the inner regions of galaxies (Sofue et al. 1986). In this model, the LOS magnetic field reverses with the sign of galactic longitude at a particular galactocentric radius (r). Reversals of the magnetic field as a function of galactocentric radius are also predicted by this model. Since both these predictions are inconsistent with the data (Fig. 3), the ring model is not applicable in this region.

(iii) Bisymmetric spiral model:

Simard-Normandin \& Kronberg (1980) proposed this model (see also Han et al. 1999) to account for the reversals of magnetic 
fields with galactocentric distances in the Galaxy. A schematic diagram of this model is shown in Fig. 5. It predicts a positive RMs towards $l=0^{\circ}$, which is what is observed. Therefore, the prediction from this model near the GC is consistent with our observations.

(iv) Another plausible configuration of the magnetic field: Magnetic field lines are typically observed to be aligned with large-scale structures in the Galaxy and beyond. In the central few kpc region of the Galaxy a bar-like distribution of matter has been suspected for a long time, and recent Spitzer observations suggest it is oriented at an angle of $44^{\circ}$ with respect to our LOS (Churchwell \& Glimpse Team 2005). An impression of this from the top of our Galaxy is shown in Fig. $6^{1}$. A bar in gas distribution in the central region of the Galaxy has also been claimed (Sawada et al. 2004). If the magnetic field lines are oriented along this bar and have a component towards us, then this could explain the positive RMs observed in all the four quadrants. We note a decrease in averaged RM near $l=0^{\circ}$ (Fig. 3). Magnetic fields in the GC region are very likely anchored to the dense molecular clouds, and within $\sim 1^{\circ}$ of the GC, they have large random motions, which reduces the magnetic field averaged over the $2.4^{\circ}$ bin centred on the GC.

\subsubsection{Random component of magnetic field}

In this section, we discuss the power spectrum of the magnetoionic ISM and then estimate the strength of the magnetic field responsible for it. Small-scale variations in a magnetoionic medium are likely to be related to electron density fluctuations in ISM, which have been studied through scattering and scintillation observations (Rickett 1990). The power spectrum of electron density irregularities is expressed by

$P(q)=C_{n}^{2} q^{-\alpha}, q_{0}<q<q_{i}$.

Where, $q$ is spatial wavenumber, and $\alpha$ is spectral index (Rickett 1977). $C_{n}^{2}$ is normalisation constant of the electron density power spectrum. The quantities $q_{0}$ and $q_{i}$ represent wavenumbers corresponding to "outer scale" and "inner scale" of the turbulence respectively.

Assuming the fluctuations in electron density and magnetic field to be zero mean isotropic Gaussian random processes with the same outer scale $\left(l_{0}\right)$, Minter \& Spangler (1996) derived $D_{\mathrm{RM}} \propto(\Delta \theta)^{\alpha-2}$. For three dimensional Kolmogorov turbulence $\alpha=11 / 3$, and $D_{\mathrm{RM}} \propto(\Delta \theta)^{5 / 3}$. However, from Fig. 2, we find the structure function is well fitted by a power law of index $0.7 \pm 0.1$ (Sect. 2.1.1) up to about $0.7^{\circ}$ and then it gets saturated. This is consistent with $D_{\mathrm{RM}} \propto(\Delta \theta)^{2 / 3}$, which would indicate $\alpha=8 / 3$, expected from two-dimensional Kolmogorov turbulence. Twodimensional turbulence results if the screen responsible for it is confined in thin sheets in the sky plane. Minter \& Spangler (1996) have found in their data that the structure function slope changes from about $5 / 3$ to $2 / 3$ at a length scale of about 7 pc. However, in our data we do not observe any significant deviation from the fit at the smallest angular separation in Fig. 2 near $0.002^{\circ}$ corresponding to a linear scale of $0.3 \mathrm{pc}$ at a distance of the GC. This will indicate if the turbulence is indeed Gaussian in nature, the thickness of the screen/screens is $\leq 0.3 \mathrm{pc}$ in the central kpc of the Galaxy. On the other hand, turbulent processes could be non Gaussian. Boldyrev \& Gwinn (2005) have shown that the Levy distribution of irregularities in a three-dimensional

\footnotetext{
${ }^{1}$ http://www.spitzer.caltech.edu/Media/mediaimages/ sig/sig05-010.shtml
}

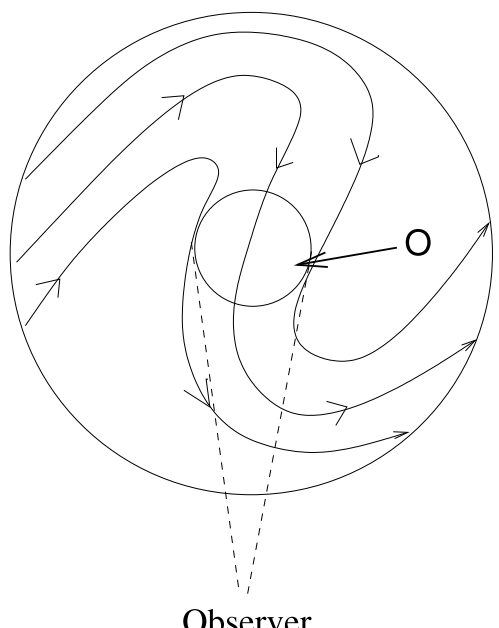

Fig. 5. A schematic diagram of the bisymmetric spiral structure of magnetic fields.

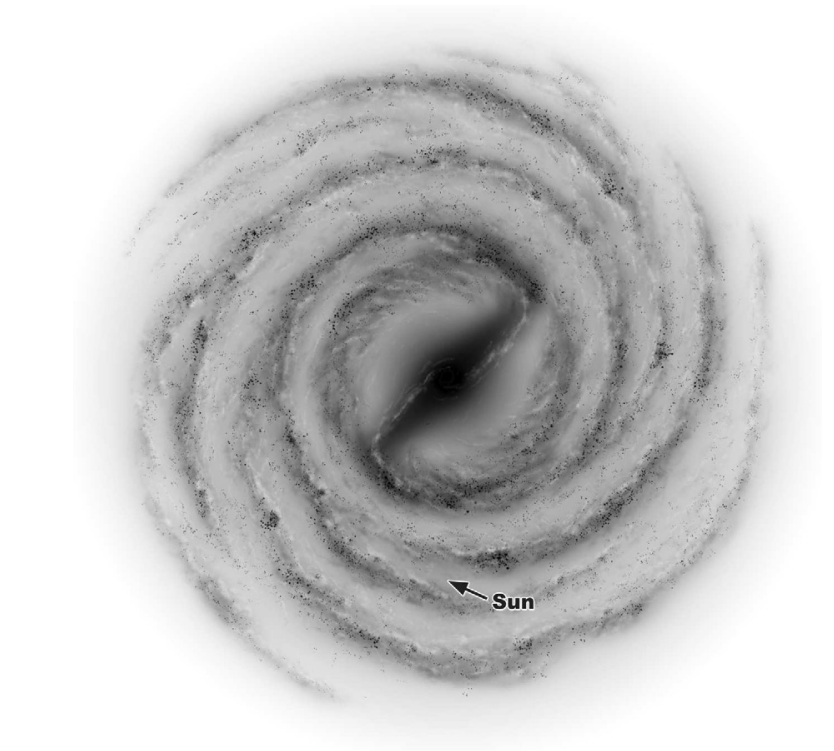

Fig. 6. A schematic view of the Galaxy from the top of the Galactic plane. Notice the kpc scale bar within the central $4 \mathrm{kpc}$ from the GC.

screen could explain the shapes and the the scaling of observational pulse profile of a pulsar. A physical realisation of such a Faraday screen is random discontinuity in the distribution of electron density and magnetic field. This process has a divergent second moment and could explain results that otherwise would require the turbulence to be two-dimensional if Gaussian random process is assumed.

To estimate the strength of the random magnetic fields, we assume the RMs to be correlated within the outer scale of the RM structure function ( $40 \mathrm{pc}$ ) (henceforth called cells). It is quite easy to show that along our LOS

$D_{\mathrm{RM}}=\left\{0.8 \times\left(\Delta n_{\mathrm{e}} \times\left\langle B_{\|}\right\rangle+n_{\mathrm{e}} \times \Delta B_{\|}\right) \times l_{0} \times \sqrt{n}\right\}^{2}$

where $\Delta n_{\mathrm{e}}$ and $\Delta B_{\|}$correspond to the fluctuating component of the electron density and magnetic fields along our LOS respectively. In the above equation, $l_{0}$ is the size of each cell and " $n$ " the number of such cells along each LOS. As discussed in Sect. 3.2.1, $n_{\mathrm{e}}$ is estimated to be about $0.4 \mathrm{~cm}^{-} 3$, and $\Delta n_{\mathrm{e}}$ is 
also believed to be about the same. In Sect. 3.2.1, $\left\langle B_{\|}\right\rangle$is estimated to be $0.6 \mu \mathrm{G}$. If the central $2 \mathrm{kpc}$ region is believed to be responsible for the observed RMs, then there will be about 50 cells along each LOS. The estimated random magnetic fields at length scales of $40 \mathrm{pc}$ corresponding to the RM structure function of $3.7 \times 10^{5} \mathrm{rad} \mathrm{m}^{-2}$ (Fig. 2) is $6 \mu \mathrm{G}$. We note that electron density distribution is quite clumpy in the inner Galaxy (Cordes et al. 1985). Therefore, the number of such cells could be much less, such that the total dispersion measure remains almost the same. In that case, $\Delta B_{\|}$would be given by $\sim 6 \times \sqrt{(n / 50)} \mu \mathrm{G}$.

\subsection{Implications for the GC magnetic field}

In previous sections we have estimated an average LOS systematic magnetic field of $\sim 1 \mu \mathrm{G}$ and a random field of $6 \mu \mathrm{G}$. However, this does not address the overall magnetic field in the central one degree from the GC, which is described below.

The observed magnetic fields in galaxies are rarely systematic. This is due to turbulence, and the random component has a field strength that is about the same in magnitude as the systematic field (Zweibel \& Heiles 1997). In the GC region, a highly turbulent magnetoionic media causes high scatter broadening of extragalactic sources. Here we estimate the strength of this random component in this region from our data, which will provide an estimate of the strength of the systematic field. As shown in Sect. 2.1.1, the outer scale of RM of sources in this region is about $40 \mathrm{pc}$, and we follow the same approach as in the previous section for calculating the random magnetic fields. There are about 7 cells within a region of angular radius $1^{\circ}$ corresponding to a linear size of about $300 \mathrm{pc}$ at a distance of $8.0 \mathrm{kpc}$. With an electron density of $0.4 \mathrm{~cm}^{-3}$ in the region, if there is a net LOS magnetic field of 1 milliGauss over a size scale equivalent to the size of these cells, this region would introduce a RM of $\sim 12800 \mathrm{rad} \mathrm{m}^{-2}$. As the magnetic fields in these cells are uncorrelated, the mean value of RMs towards sources could be small, but the rms value of RMs along different LOSs would be $\sim 34000 \mathrm{rad} \mathrm{m}^{-2}$. There are 6 source components in our sample seen through the central $1.1^{\circ}$ from the GC, but we do not find any of their absolute RMs to be significantly higher than the mean RM from the whole sample. The estimated rms RM from our sample is consistent with a random field of $\sim 20 \mu \mathrm{G}$ in this central $300 \mathrm{pc}$ region of the Galaxy. This suggests that the strong magnetic fields near the NTFs could only be a local enhancement to the GC magnetic fields and does not fill the entire $300 \mathrm{pc}$ region.

This outer scale is much larger than the measured size scale of the Faraday screen of $\sim 10^{\prime \prime}(0.4 \mathrm{pc})$ towards the GC NTFs G359.54+0.18 (Yusef-Zadeh et al. 1997) and Snake (G359.1-0.2) (Gray et al. 1995), and is consistent with the size scale of low-density HII regions in the Galaxy (Anantharamaiah 1985). This is also close to the turbulence scale expected from supernova explosions in the GC (Boldyrev \& Yusef-Zadeh 2006). The difference of two order of magnitude in the size scale of turbulence in the Faraday RMs towards the background sources as compared to regions close to NTFs indicates the magnetoionic properties of ISM in the GC region is vastly different than what is observed close to NTFs. Recent observations (LaRosa et al. 2005) and a model (Boldyrev \& Yusef-Zadeh 2006) also support this conclusion. If NTFs are dynamic structures, local enhancement of magnetic fields in their vicinity could also be explained (Shore \& Larosa 1999).

\section{Conclusions}

To study the properties of the Faraday screen near the GC, we measured RMs towards 60 background extragalactic sources through the $-6^{\circ}<l<6^{\circ},-2^{\circ}<b<2^{\circ}$ region of the Galaxy. To our knowledge, this provides the first direct determination of large-scale magnetoionic properties of the central $1 \mathrm{kpc}$ region of the Galaxy not biased by NTF environments. We find a large-scale LOS magnetic fields that point towards us. Either the bisymmetric spiral model of magnetic field in the Galaxy or the magnetic-field lines that are mostly aligned with the central bar of the Galaxy could explain a largely positive RM in the central $1 \mathrm{kpc}$ of the Galaxy. This large-scale magnetic field has a lower limit of $0.6 \mu \mathrm{G}$ along the LOS. The outer scale of the $\mathrm{RM}$ structure function is about $40 \mathrm{pc}$. The RM structure function is well-fitted with a power law index of $0.7 \pm 0.1$ at length scales of 0.3 to $100 \mathrm{pc}$ at the distance of the GC, which is inconsistent with a three dimensional Kolmogorov turbulence. A magnetic field fluctuation of $\sim 6 \mu \mathrm{G}$ along with electron density fluctuation could explain the observed RM structure function in the central $1 \mathrm{kpc}$ of the Galaxy. However, in the inner $300 \mathrm{pc}$, the maximum random component of the magnetic field is estimated to be $\sim 20 \mu \mathrm{G}$. Since GC region has a highly turbulent ISM, this random magnetic field is very likely have a similar strength to the systematic field. The observed outer scale of the magnetoionic medium in this region also does not appear to be less than what is determined from the whole sample $(\sim 40 \mathrm{pc})$. This is much larger than the scale size of the RM structure function $\sim 10^{\prime \prime}(0.4 \mathrm{pc})$ observed near the NTFs in the GC. This indicates that properties of the Faraday screen in the GC is very different from what is found close to the NTFs. The milliGauss magnetic fields estimated near the NTFs are localised and do not pervade the central $300 \mathrm{pc}$ of the Galaxy. A more detailed investigation of the magnetic field involving background sources several times more than the present study would, however, be required to make a model of the magnetic field configuration in the region.

Acknowledgements. We thank Rajaram Nityananda for introducing the Bootstrap technique to us. We also thank the anonymous referee whose comments helped to improve the quality of the paper.

\section{References}

Anantharamaiah, K. R. 1985, J. Astrophys. Astron., 6, 203

Anantharamaiah, K. R., Pedlar, A., Ekers, R. D., \& Goss, W. M. 1991, MNRAS, 249,262

Efron, B. 1979, Ann. Statistics, 7, 1

Beck, R., Brandenburg, A., Moss, D., Shukurov, A., \& Sokoloff, D. 1996, ARA\&A, 34, 155

Boldyrev, S., \& Gwinn, C. R. 2005, ApJ, 624, 213

Boldyrev, S., \& Yusef-Zadeh, F. 2006, ApJ, 637, L101

Bower, G. C., Backer, D. C., \& Sramek, R. A. 2001, ApJ, 558, 127

Brown, J. C., Haverkorn, M., Gaensler, B. M., et al. 2007, ApJ, 663, 258

Churchwell, E., \& Glimpse Team. 2005, in Rev. Mex. Astron. Astrofis. Conf. Ser., ed. S. Torres-Peimbert, \& G. MacAlpine, 53

Clegg, A. W., Cordes, J. M., Simonetti, J. M., \& Kulkarni, S. R. 1992, ApJ, 386, 143

Cordes, J. M., \& Lazio, T. J. W. 2002 [arXiv:astro-ph/0207156]

Cordes, J. M., Weisberg, J. M., \& Boriakoff, V. 1985, ApJ, 288, 221

Davidson, J. A. 1996, in Polarimetry of the Interstellar Medium, ASP Conf. Ser., 97, 504

Frick, P., Stepanov, R., Shukurov, A., \& Sokoloff, D. 2001, MNRAS, 325, 649

Gray, A. D., Nicholls, J., Ekers, R. D., \& Cram, L. E. 1995, ApJ, 448, 164

Han, J. L., \& Qiao, G. J. 1994, A\&A, 288, 759

Han, J. L., Manchester, R. N., \& Qiao, G. J. 1999, MNRAS, 306, 371

Killeen, N. E. B., Lo, K. Y., \& Crutcher, R. 1992, ApJ, 385, 585

Krause, F. 1987, in Interstellar Magnetic Fields: Observation and Theory, 8

Lang, C. C., Anantharamaiah, K. R., Kassim, N. E., \& Lazio, T. J. W. 1999a, ApJ, 521, L41

Lang, C. C., Morris, M., \& Echevarria, L. 1999b, ApJ, 526, 727 
LaRosa, T. N., Brogan, C. L., Shore, S. N., et al. 2005, ApJ, 626, L23

Lazio, T. J. W., \& Cordes, J. M. 1998, ApJ, 505, 715

Lazio, T. J. W., Anantharamaiah, K. R., Goss, W. M., Kassim, N. E., \& Cordes, J. M. 1999, ApJ, 515, 196

Manchester, R. N., Hobbs, G. B., Teoh, A., \& Hobbs, M. 2005, AJ, 129, 1993

Minter, A. H., \& Spangler, S. R. 1996, ApJ, 458, 194

Mitra, D., Wielebinski, R., Kramer, M., \& Jessner, A. 2003, A\&A, 398, 993

Morris, M. 1998, in The Central Regions of the Galaxy and Galaxies, ed. Y. Sofue, IAU Symp., 184, 331

Nord, M. E., Lazio, T. J. W., Kassim, N. E., et al. 2004, AJ, 128, 1646

Novak, G., Chuss, D. T., Renbarger, T., et al. 2003, ApJ, 583, L83

Rand, R. J., \& Kulkarni, S. R. 1989, ApJ, 343, 760

Rand, R. J., \& Lyne, A. G. 1994, MNRAS, 268, 497

Rickett, B. J. 1977, ARA\&A, 15, 479

Rickett, B. J. 1988, in Radio Wave Scattering in the Interstellar Medium, AIP Conf. Proc., 174, 2

Rickett, B. J. 1990, ARA\&A, 28, 561

Roy, S. 2004, Bull. Astron. Soc. India, 32, 205

Roy, S., Pramesh Rao, A., \& Subrahmanyan, R. 2003, Astron. Nachr. Suppl., 324,41
Roy, S., Rao, A. P., \& Subrahmanyan, R. 2005, MNRAS, 360, 1305

Sawada, T., Hasegawa, T., Handa, T., \& Cohen, R. J. 2004, MNRAS, 349, 1167

Schwarz, U. J., \& Lasenby, J. 1990, in Galactic and Intergalactic Magnetic Fields, IAU Symp., 140, 383

Shore, S. N., \& Larosa, T. N. 1999, ApJ, 521, 587

Simard-Normandin, M., \& Kronberg, P. P. 1980, ApJ, 242, 74

Sofue, Y., \& Handa, T. 1984, Nature, 310, 568

Sofue, Y., Fujimoto, M., \& Wielebinski, R. 1986, ARA\&A, 24, 459

Taylor, J. H., \& Cordes, J. M. 1993, ApJ, 411, 674

Uchida, Y., Sofue, Y., \& Shibata, K. 1985, Nature, 317, 699

van Langevelde, H. J., Frail, D. A., Cordes, J. M., \& Diamond, P. J. 1992, ApJ, 396, 686

Yusef-Zadeh, F., \& Morris, M. 1987a, AJ, 94, 1178

Yusef-Zadeh, F., \& Morris, M. 1987b, ApJ, 322, 721

Yusef-Zadeh, F., Roberts, D. A., Goss, W. M., Frail, D. A., \& Green, A. J. 1996, ApJ, 466, L25

Yusef-Zadeh, F., Wardle, M., \& Parastaran, P. 1997, ApJ, 475, L119

Yusef-Zadeh, F., Roberts, D. A., Goss, W. M., Frail, D. A., \& Green, A. J. 1999, ApJ, 512, 230

Zweibel, E. G., \& Heiles, C. 1997, Nature, 385, 131 